\title{
Analysis of Image Inpainting Techniques with Exemplar, Poisson, Successive Elimination and 8 Pixel Neighborhood Methods
}

\author{
Muthukumar S \\ CITE Department, MS University, \\ Tirunelveli, Tamil Nadu, India
}

\author{
Dr.Krishnan .N \\ CITE Department, MS University, \\ Tirunelveli, Tamil Nadu, India
}

\author{
Pasupathi.P, Deepa. S \\ CITE Department, MS University, \\ Tirunelveli, Tamil Nadu, India
}

\begin{abstract}
This paper discusses removing large objects from digital images and fills the hole that is left behind in a visually plausible way. We present a novel and efficient algorithm that fills the hole by exemplar-based synthesis. Here the simultaneous propagation of texture and structure information is achieved by a single, efficient algorithm. The texture image is repaired by the exemplar -based method; for the structure image, the Laplacian operator is employed to enhance the structure information, and the Laplacian image is inpainted by the exemplar-based algorithm, followed by a reconstruction based on the Poisson equation. To improve the computational efficiency of our algorithm we go for successive elimination algorithm (SEA). In 8 pixel neighborhood method, identifying central pixel value by investigating surrounded 8 neighborhood pixel properties like color variation, repetition, intensity and direction. Finally we compare speed and accuracy of a picture enhancement using 8 pixel neighborhood with exemplar based poisson \& successive elimination method
\end{abstract}

\section{Keywords}

Image restoration, Object removal, Image Inpainting, texture synthesis, Structure propagation, SEA algorithm

\section{INTRODUCTION}

In real world, many people need a system to recover the damaged photographs, artwork, designs, drawings etc..Damage may be due to various reasons like scratches, overlaid text or graphics, scaled image etc., This system could enhance and return a good looking photograph using a technique called inpainting or retouching. The observer does not know the original image. Traditionally, inpainting has been done by professional artists. But we could not expect the accuracy and quality if it was done by human and time consuming process.

The objective of inpainting is to reconstitute the missing or damaged portions of the work, in order to make it more legible and to restore its unity. The need to retouch the image in an unobtrusive way extended naturally from paintings to photography and film. Digital techniques are ranging from attempts to fully automatic detection and removal of scratches in film, all the way to software tools that allow a sophisticated but mostly manual process.

In this article, we compared the following algorithms of image inpainting in terms of efficiency, accuracy and time consumption.
Object removal by exemplar based inpainting method

- Poison Equation method

- Successive elimination method

- 8-pixel neighborhood fast sweeping method .

\section{EXEMPLAR-BASED REGION FILLING}

The user selects a target region, $\Omega$, to be removed and filled. The source region, $\Phi$, may be defined as the entire image minus the target region $(\Phi=\mathrm{I}-\Omega)$, as a dilated band around the target region, or it may be manually specified by the user.

Our algorithm iterates the following three steps until all pixels have been filled:

- $\quad$ Computing patch priorities

- $\quad$ Texture .synthesis

- $\quad$ Filling order

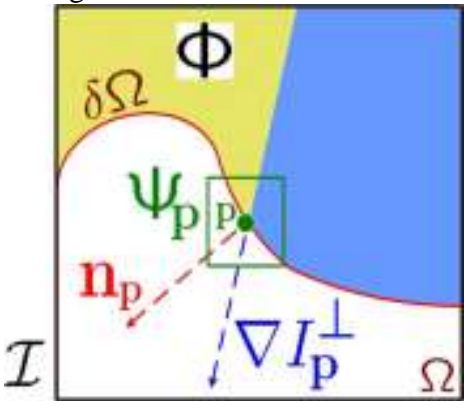

Figure 1 Notation Diagram

\subsection{Computing patch priorities.}

Our algorithm performs the synthesis task through a best-first filling strategy that depends entirely on the priority values that are assigned to each patch on the fill front. The priority computation is biased toward those patches which: (i) are on the continuation of strong edges and (ii) are surrounded by highconfidence pixels. Given a patch $\mathrm{p}$ centred at the point $\mathrm{p}$ for some $\mathrm{p} \in \delta \mathrm{n}$, we define its priority $\mathrm{P}(\mathrm{p})$ as the product of two terms:

$\mathrm{P}(\mathrm{p})=\mathrm{C}(\mathrm{p}) \mathrm{D}(\mathrm{p})$

$\mathrm{C}(\mathrm{p})$ the confidence term and $\mathrm{D}(\mathrm{p})$ the data term, 


$$
\begin{aligned}
& c(p)=\left(\sum q \in \Psi_{p} \cap(I-\Omega) c(q)\right) /\left|\Psi_{p}\right| \\
& D(p)=\left(\mid \nabla I \perp p . n_{p}\right) \mid / \alpha
\end{aligned}
$$

The confidence term $C(p)$ may be thought of as a measure of the amount of reliable information surrounding the pixel $\mathrm{p}$. The intention is to fill first those patches which have more of their pixels already filled, with additional preference given to pixels that were filled early on (or that were never part of the target region).

Patches that include corners and thin tendrils of the target region will tend to be filled first, as they are surrounded by more pixels from the original image. These patches provide more reliable information against which to match. Conversely, patches at the tip of "peninsulas" of filled pixels jutting into the target region will tend to be set aside until more of the surrounding pixels are filled in.

\subsection{Texture Synthesis}

Once all priorities on the fill front have been computed, the patch $\mathrm{p}$ with highest priority is computed. We then fill it with data extracted from the source region. In traditional inpainting techniques, pixel-value information is propagated via diffusion. As noted previously, diffusion necessarily leads to image smoothing, which results in blurry fill-in, especially of large regions. On the contrary, we propagate image texture by direct sampling of the source region. We search in the source region for that patch which is most similar to $\mathrm{p}$.

Formally,

$$
\begin{aligned}
& \Psi^{\prime} q=\arg \min d\left(\Psi^{\prime} p, \Psi^{\prime} q\right) \\
& \Psi^{\prime} q \in \Phi
\end{aligned}
$$

\subsection{Filling order}

Exemplar-based filling may be capable of propagating both texture and structure information. This section demonstrates that the quality of the output image synthesis is highly influenced by the order in which the filling process proceeds. As it can be observed, the ordering of the filled patches produces the horizontal boundary between the background image regions to be unexpectedly reconstructed as a curve.

A concentric-layer ordering, coupled with a patch-based filling may produce further artefacts. Another desired property of a good filling algorithm is that of avoiding "over-shooting" artefacts that occur when image edges are allowed to grow indefinitely.

\section{Region Filling Algorithm}

1. Find all boundary point of cracked portion
2. Find data pattern \& confident for each
boundary point
3. Multiply data pattern \& confident of each
Boundary point (It is total

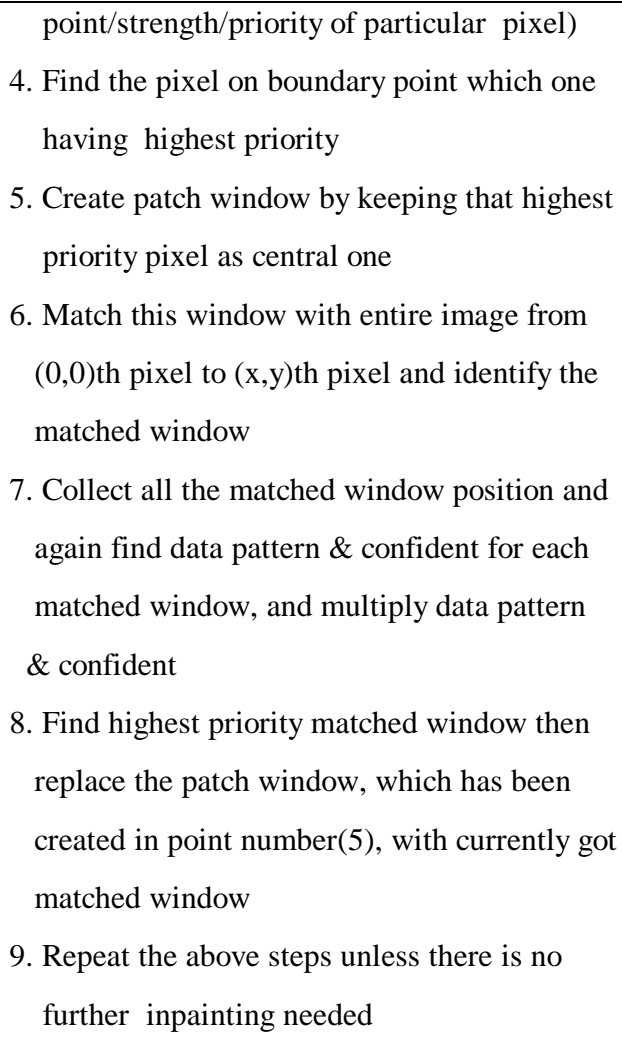

7. Collect all the matched window position and again find data pattern \& confident for each matched window, and multiply data pattern $\&$ confident

8 . Find highest priority matched window then replace the patch window, which has been created in point number(5), with currently got matched window

9. Repeat the above steps unless there is no further inpainting needed

\section{3 .POISSON EQUATION}

First we decompose the image into two components. The structure and texture. And then repair the two components separately according to their image characteristics. The texture Image is repaired by the exemplar based method. The structure image, Laplacian operator is employed to enhance the structure information and the Laplacian image is inpainted by the exemplar based algorithm followed by a reconstruction based on the Poisson equation.

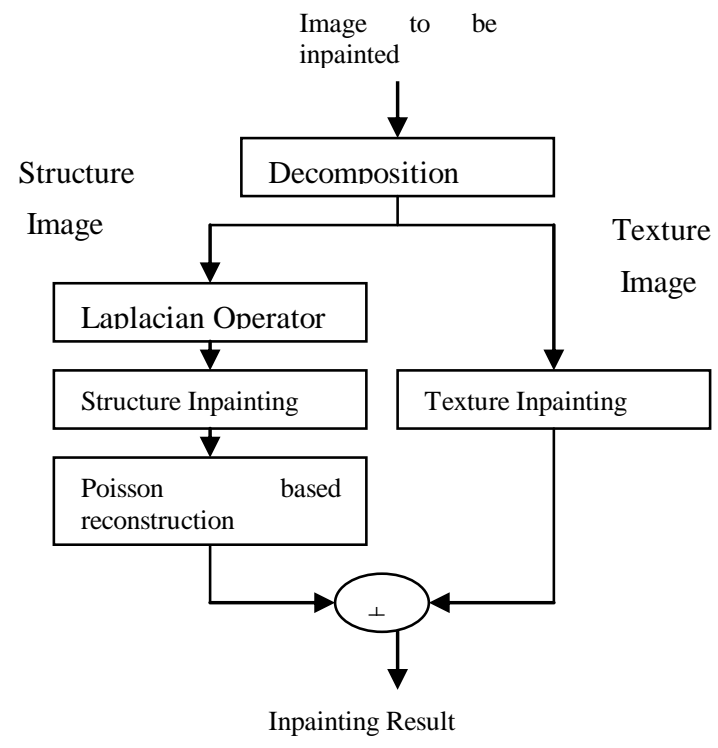

Figure 2 Inpainting flowchart 
In most existing inpainting methods, the inpainting domain is assumed as the smoothest continuation of the local structure, where smoothness can be defined in different ways. However, these assumptions become inappropriate when the size of the inpainting domain increases or there is texture inside the hole. Moreover, these methods only employ a small ring area surrounding the inpainting domain to repair the missing part, which makes the algorithms sensitive to noise and inappropriate for inpainting large holes. Moreover, if the inpainting domain includes both texture and structure, the texture will disturb the inpainting process of the structure, which causes false edges in the repaired image.

Poisson Algorithm

\section{Find the portion need to be inpainted.}

2. Split image into two components as structure and texture.

3. Do process as same as exemplar for texture image.

4. Do smoothing process for structure image-

Laplacian operator.

5.Combined both output produced by step $3 \& 4$

6. Result would be inpainted image.

\section{IMAGE INPAINTING BASED ON SUCCESSIVE ELIMINATION}

To improve the efficiency in Exemplar method successive elimination algorithm( SEA) is employed to obtain global optimal solution. The basic idea is to obtain the best estimate of the vectors by successively eliminating the search positions in the search window and thus decreasing the number of matching evaluations that require very intensive computations.

The computational efficiency is increased by choosing the successive elimination algorithm which is based on sum of absolute differences ( $\mathrm{SAD}$ ) to obtain the global optimal solution, and the definition of SAD is as follows:

$$
d(\Psi p, \Psi q)=\sum_{i=0}^{N-1} \sum_{j=0}^{N-1}|f(x+i, y+j)-f(m+i, n+j)|
$$$$
M(m, n)=\sum_{i=0}^{N-1} \sum_{j=0}^{N-1}|f(m+i, n+j)|
$$

$$
R=\sum_{i=0}^{N-1} \sum_{j=0}^{N-1}|f(x+i, y+j)|
$$

That implies

$$
R-S A D(u, v) \leq M(m, n) \leq R+S A D(u, v)
$$

Successive Elimination Algorithm

1. Find all boundary point of cracked portion

2. Find data pattern \& confident for each

boundary point

3. Multiply data pattern \& confident of each

boundary point (It is total

point/strength/priority of particular pixel)

4. Find the pixel on boundary point which one having highest priority

5. Create patch window by keeping that highest priority pixel as central one

6. Match this window with entire image from $(0,0)$ th pixel to $(x, y)$ th pixel and identify the matched window

7. Stop the process of fetching another match window once you got first match window (In exemplar we will find all the possible match window then we do finding high priority for matched window then we choose the best window, but in SEA if one window matched means it will not fetch another match window)

8. Replace the patch window, which has been created in point number (5), with currently got matched window

9. Repeat the above steps unless there is no further inpainting needed

\section{IMAGE INPAINTING BASED ON 8 PIXEL NEIGHBORHOOD}

The neighbourhood of a pixel is the set of pixels that touch the neighbourhood of a pixel can have a maximum of 8 pixels (images are always considered 2D). The 8 pixel neighborhood of a pixel, $\mathrm{P}$, is the set of 8 pixels which share a vertex or edge with that pixel. These pixels are namely pixels P1, P2, P3, P4, P5, P6, P7 and P8 shown in Figure 3 below.

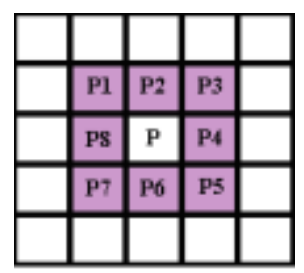

Figure 3 Neighbors 
Crimimisi, in exemplar method proposed a repair method based on the formation of the texture, filling the blank areas by choosing suitable texture on the border of the region to be repaired by block matching methods, it has a better effect to the texture inpainting, but it has worse effect to structural repair.

These methods above can be achieved good results in a certain type of image, but there are obvious limitations, that is not suit for each case and most of them are based on iterative formula, one point restoration often need thousands of iteration, running a long time and repair one image it has to cost 3 or 4 minutes to complete, which limits their promotion and application. In light of this situation, in this paper, we propose a new algorithm based on the 8-neighborhood fast sweeping method on the basis of maintain the effectiveness of rehabilitation to shorten repair time greatly.

\section{EXPERIMENTAL RESULTS}

We apply algorithm to both the object removal and damaged frames in old films. The experiment results show that the inpainted images are visually pleasant and computational efficiency is improved in Successive Elimination Method.

Exemplar Method works well for large objects. For single dimensions like line, Arc then Poisson method is useful.

Finally we compare speed and accuracy of a picture enhancement using 8 pixel neighborhood, if the picture is like natural scenario then 8 pixel is good for computation speed and accuracy than Exemplar method .

Table 1 Inpainting -Computational Cost Analysis

\begin{tabular}{|c|c|c|c|c|}
\hline & \multicolumn{4}{|c|}{ The computing cost of } \\
\cline { 2 - 5 } & $\begin{array}{c}\text { Exe } \\
\text { mpl } \\
\text { ar }\end{array}$ & Poission & $\begin{array}{c}\text { Successi } \\
\text { ve } \\
\text { Eliminat } \\
\text { ion }\end{array}$ & $\begin{array}{c}\text { 8 Pixel } \\
\text { neighbo } \\
\text { rhood }\end{array}$ \\
\hline Natural & 461 & $583 \mathrm{sec}$ & $421 \mathrm{sec}$ & $49 \mathrm{sec}$ \\
Scenery 1 & $\mathrm{sec}$ & & $272 \mathrm{sec}$ & $32 \mathrm{sec}$ \\
\hline Natural & 329 & $426 \mathrm{sec}$ & & $47 \mathrm{sec}$ \\
Scenery 2 & $\mathrm{sec}$ & & $126 \mathrm{sec}$ & \\
\hline Batch & 239 & $302 \mathrm{sec}$ & $129 \mathrm{sec}$ & $39 \mathrm{sec}$ \\
Filling 1 & $\mathrm{sec}$ & & & \\
\hline Batch & 185 & $270 \mathrm{sec}$ & & \\
Filling 2 & $\mathrm{sec}$ & & &
\end{tabular}

If the sample picture is batch filling type then Exemplar method is good in computation time and accuracy than 8 pixel neighborhood method.

\section{CONCLUSION}

This paper has presented different algorithms for removing objects and inpainting damaged images. The result is an image in which the selected object has been replaced by a visually plausible background that mimics the appearance of the source region. Our approach employs an exemplar-based texture synthesis. To improve the efficiency of filling one dimension and two dimension objects Poisson method is best. To improve the computational cost successive elimination algorithm is employed to obtain global optimal solution for 2 dimension.

In 8 pixel -neighborhood fast sweeping method experimental result shows that there is substantive increase in the rate of image inpainting for small region ie. One dimension and blur in large region.

\section{REFERENCES}

[1] Yu Jing, Su Kai-na. "A Survey of Block-based Motion Estimation" Journal of Image and Graphics, vol. 12, Dec, 2007, pp. 2031-2041.

[2] Li Guo-yu, Fu Yu-zhuo, Wang Shiming. “An Improved Multilevel Successive Elimination Algorithm." Computer Simulation, vol. 22, Sep, 2005,pp. 89-91.

[3] Huang Shuai, Song Guo-xin. "Hexagon-based Self-adaptive Search Algorithm Using Successive Elimination." Computer Engineering, vol. 32, 2006, pp. 212-214.

[4] Jiao Rong-hui, Guo Li, Guo Li-sheng. "An Improved Motion Estimation Algorithm." Microelectronics \& Computer, vol. 23, Dec, 2006, pp. 213-215.

[5] W. Li, and E. Salari. "Successive elimination algorithm for motion estimation". IEEE Transaction on Image Processing, vol, 4, Jan, 1995, pp. 105-107.

[6] X. Q. Gao, C. J. Duanmu, C. R. Zou and Z. Y. He. "Multilevel successive elimination algorithm for motion estimation in video coding." in Proc. IEEE International Symposium. Circuits and Systems, vol. 4, 1999, pp. 227-230.

[7] An Image Inpainting Approach Based on thePoisson Equation, Xiaowei Shao, Zhengkai Liu, Houqiang Li. Department of Electronic Engineering and Information Science, University of Science and Technology.

[8] An Image Inpainting Technique Based on 8-Neighborhood Fast Sweeping Method. Jing Xu1, Daming Feng2, Jian Wu1, Zhiming Cui11. The Institute of Intelligent Information Processing and Application, Soochow University, Jiangsu, 215006, China. School of Computer S\&T, Soochow University, Jiangsu Province.

[9] Criminisi, P. Pérez, and K. Toyama. "Object removal by exemplar-based inpainting." in Proc. Conf. Computer Vision and Pattern Recognition, Madison, WI, June 2003.

[10] Criminisi, P. Pérez, and K. Toyama. "Region Filling and Object Removal by Exemplar-Based Image Inpainting." IEEE Transaction on Image Processing, vol. 13, Sep, 2004, pp. 1200-1212. 